\title{
Clinical Profile of Invasive Pneumococcal Disease in Patan Hospital, Nepal
}

\author{
Chhetri UD, Shrestha S, Pradhan R, Shrestha A, Adhikari N, Thorson S, Pollard AJ, Murdoch \\ DR, Kelly DF
}

Department of Pedriatrics

Patan Academy of Health Sciences (PAHS),

Patan Hospital, Lagankhel, Nepal.

\section{Corresponding Author}

Dr Uma Devi Chhetri

Department of Pediatrics

Patan Academy of Health Sciences,

Patan Hospital, Lagankhel, Nepal

Email: udchhetri@yahoo.com

\section{Citation}

Chhetri U D, Shrestha S, Pradhan R, Shrestha A, Adhikari N, Thorson S, Pollard A J, Murdoch D R, Kelly D F. Clinical Profile of Invasive Pneumococcal Disease in Patan Hospital, Nepal. Kathmandu Univ Med J 2011;33(1)45-9.

\section{ABSTRACT \\ Background}

Pneumococcal infection is one of the leading causes of pneumonia, meningitis and septicemia in developing countries. It accounts for one million deaths each year in children.

\section{Objectives}

The objective of this study is to see the clinical profile of invasive pneumococcal disease, antibiotics sensitivity pattern and prevalent serotypes in children admitted at Patan Hospital.

\section{Methods}

This is a retrospective analytical study conducted in the department of Paediatrics, Patan hospital. The lab data of those children who grew pneumococci in their blood, cerebrospinal fluid or body fluids over a period of 3 years (January 2007 to Dec 2009) were collected and the case files were then studied.

\section{Results}

Out of 42 cases of invasive pneumococcal diseases studied admitted diagnoses included pneumonia, febrile seizure, bacteremia or septicemia, meningitis, acute gastroenteritis and glomerulonephritis. Twenty seven of them were children under five. The male to female ratio was 1.7:1. On investigation $64 \%, 52 \%$ and $5 \%$ of the patients had leucocytosis, anaemia, and leucopenia respectively. Twenty six of them had radiological changes suggestive of pneumonia. Streptococcus pneumoniae grew in 38 blood samples, 5 cerebrospinal fluid and 3 pleural fluids. Almost all of these isolates were sensitive to penicillin, cefotaxime, amoxycillin, choloramphenicol, erythromycin and ofloxacin and resistant to cotrimoxazole and gentamicin.Pneumococcal serotypes found in our study were 1, 14, 5, 23B, 6B, 8, 9A, 9V, 10A, 15 and 23F (11 serotypes).

\section{Conclusions}

Penicillin is still the most effective antibiotic for streptococcal infection in our study. Of the pneumococcal serotypes identified; $36 \%$ were covered by the 7 -valent pneumococcal conjugate vaccine, $54 \%$ each by PCV-10 and PCV-13, and $72 \%$ by the e 23 valent vaccines.

\section{KEY WORDS}

Invasive Pneumococcal Disease, Serotypes, Streptococcus pneumoniae, 


\section{INTRODUCTION}

Pneumococcal infection is one of the leading causes of pneumonia, meningitis and septicemia in developing countries. ${ }^{1}$ More than $90 \%$ of pneumococcal pneumonia deaths in children occur in developing countries. It accounts for one million children deaths each year. ${ }^{1}$

Invasive pneumococcal disease generally defined by isolation of Streptococcus pneumoniaea from a normally sterile site (e.g. blood, CSF, but not sputum). Pneumococcal infection is most prevalent in winter season. It can cause osteomyelitis, arthritis, soft tissue swelling, early onset neonatal sepsis and otitis media. Many children may not have identifiable primary focus of infection. It may cause complications such as pleural effusion, empyema or subdural effusion.

The infection is more common between 6 months to 5 years of age, in immunocompromised children and in children with chronic disease. Other risk factors for infections are: living in a crowded environment, being under weight, having a history of any severe illness in past 6 months, exposure to smoking or passive smoking and having an upper respiratory tract infection (URTI). ${ }^{1,2}$

Laboratory diagnosis is made by a positive culture from blood, cerebrospinal fluid, pleural or other fluids, and is usually accompanied by leukocytosis and anemia.

Most respond to penicillin and the temperature returns to normal within 24 hrs of treatment. Pneumococcal vaccines are available for prevention. Strains non-susceptible to penicillin, cefotaxime and ceftriaxone have been identified in the US and worldwide.

The objectives of this study were to see the clinical profile of invasive pneumococcal disease, the antibiotic sensitivity patterns and detail the serotypes of Streptococcus pneumoniea in children admitted in Patan Hospital.

\section{METHODS}

This was an analytical study. A list of the admitted children who grew pneumococci in their blood, cerebrospinal or body fluids over a period of 3 years (Jan 2007 to Dec 2009) were collected from our laboratory and the case files were then studied. A proforma was developed to include identification, race, completed age, sex, date of admission, discharge, duration of stay, address, different clinical features, investigation, treatment, and outcome. The completed forms were then analyzed.

Weight for age was classified as normal, grade I, grade II (-2SD) and grade III (-3SD). Leucocytosis was taken as WBC count $>15000 / \mathrm{dl}$ and leucopenia as $<5000 /$ dl irrespective of age. Neutropenia was defined as mild, moderate and severe based on absolute neutrophil counts of $<1500$, $<1000$ and $<500 /$ dl respectively.

Anaemia was classified as mild for hematocrit of $<33 \mathrm{gm} /$ $\mathrm{dl}$ for less than 6 years children, $<36 \mathrm{gm} / \mathrm{dl}$ for children $>6$ years; moderate for hematocrit of $15-30 \mathrm{gm} / \mathrm{dl}$ and severe for hematocrit of $<15 \mathrm{gm} / \mathrm{dl}$.

Decreased $\mathrm{SpO} 2$ was taken as less than $92 \%$ in room air.

\section{RESULTS}

Forty two cases of invasive pneumococcal disease were admitted over a 3 yrs period (from Jan 2007 to Dec 2009) were studied. Twenty-five patients were male and seventeen were female (M:F=1.7:1). Eight (19\%) had a history of recent antibiotics taken in the previous two weeks.

Out of 42 cases, 21 cases were presented in the spring, 12 in winter five in the summer and four in autumn.

The majority of cases were from Lalitpur district (30), followed by Kathmandu (9), with one each from Makwanpur, Dhading and Gorkha.

The majority of cases were admitted from our emergency department (35) and only seven patients were admitted through the pediatric referral clinic.

Among 42 children 27 (66\%) were below five years of age and 15 (33\%) were between five to fourteen years of age.

The majority presented with fever (41), cough (26) and/ or chest pain (20).Others symptoms were chills (13), diarrhea (11), abdominal pain (7) and seizure (7).

Major presenting signs were tachypnoea (25), respiratory distress (16) and/ or a decreased SpO2 (11).

None of the patients had been vaccinated against pneumococcus.

Out of 42 patients, 24 (57\%) had normal weight for age, $15(36 \%)$ had grade I and $3(7 \%)$ had grade II under-weight respectively.

\section{INVESTIGATIONS}

None of the patients were found to have severe anaemia, while eight children had moderate and four had mild anaemia.

Leucocytosis was present in twenty-seven (64\%) patients, and two patients presented with leucopenia.

Chest $x$-rays revealed 16 with consolidation, 8 infiltrations, 13 with normal reading and 5 with para-pneumonic effusions. All patients admitted as "pneumonia" had positive $x$ rays except one.

\section{Culture and Sensitivity Report}

Out of forty-two cases Streptococcus pneumoniea were isolated in a total forty-six samples; thirty eight blood cultures, five CSF culture (one CSF alone) and three pleural fluid cultures (one pleural fluid alone).

Out of 6 meningitis cases 4 grew S. pneumoniea both in 
blood and CSF, one did not grow S. pneumoniae in CSF but did grow in the blood and one grew only in CSF. Binex test was positive in all 5 of CSFs tested. Four of the meningitis not only had both blood and CSF positive but also had positive Binex test also in CSF7. One of the meningitis was diagnosed by positive Binex test in CSF alone without growth of S. pneumoniae in CSF (blood culture was positive).

Out of 3 pleural fluid cultures, 2 grew S. pneumoniae in blood also. Binex test was positive in the only plural fluid tested.

The serotypes isolated were as shown in figure 2. Binax was positive in 6 cases ( 5 CSF and one pleural fluid).

Figure 1. Antibiotics sensitivity pattern of the isolates.

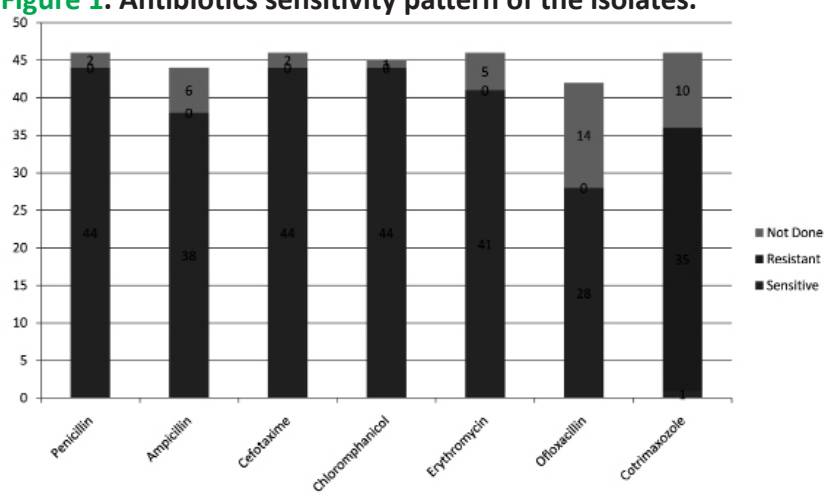

Figure 2. Pneumococcal serotypes isolated in blood, CSF and

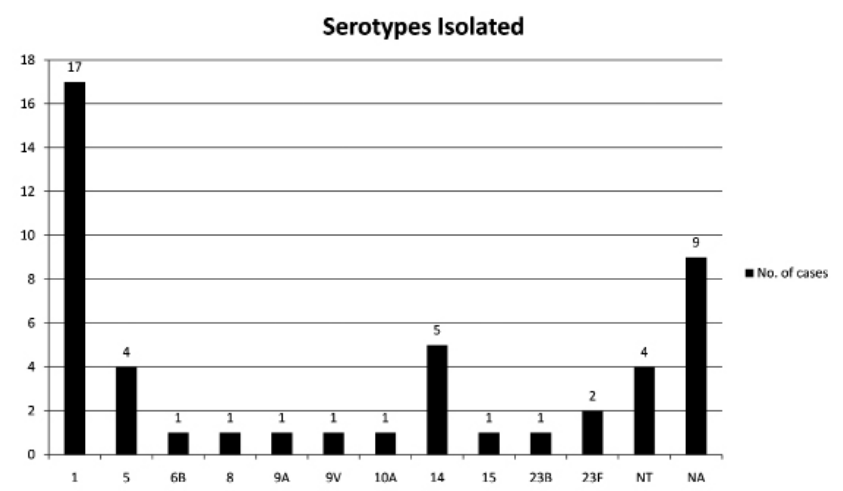

plural fluid.

$\mathrm{NT}=$ Not traceable, $\mathrm{NA}=$ Not available

Table 1. Serotypes Isolated in Other Studies. 3,4,5,6,10,12

\begin{tabular}{|lccc|}
\hline & Sensitive & Resistant & Not Done \\
\hline Penicillin & 44 & 0 & 2 \\
\hline Ampicillin & 38 & 0 & 6 \\
\hline Cefotaxime & 44 & 0 & 2 \\
\hline Chloromphanicol & 44 & 0 & 1 \\
\hline Erythromycin & 41 & 0 & 5 \\
\hline Ofloxacillin & 28 & 0 & 14 \\
\hline Cotrimaxozole & 1 & 35 & 10 \\
\hline
\end{tabular}

Table 2. Serotypes covered by different pneumococcal vaccines.

\begin{tabular}{|cc|}
\hline Sero types & No. of cases \\
\hline 1 & 17 \\
\hline 5 & 4 \\
\hline $6 B$ & 1 \\
\hline 8 & 1 \\
\hline $9 A$ & 1 \\
\hline $9 V$ & 1 \\
\hline $10 A$ & 1 \\
\hline 14 & 5 \\
\hline 15 & 1 \\
\hline $23 B$ & 1 \\
\hline $23 F$ & 2 \\
\hline$N T$ & 4 \\
\hline NA & 9 \\
\hline
\end{tabular}

\section{DIAGNOSES}

Out of forty-two cases, twenty-seven (64\%) cases were discharged as diagnosis of pneumonia, eight (19\%) as bacteremia alone or septic shock, eight (19\%) as febrile seizure with bacteremia, six (14\%) as meningitis, five (12\%) as parapneumonic effusion (12\%), and one each of (2\%) as glomerulonephritis, and diarrhea. A few cases had infection in more than one site, or more than one diagnosis.

Twelve (29\%) cases had a history of recent URTI and eight (19\%) had taken antibiotics in last 2 weeks. Complications seen were 3 pleural effusions and 2 frank empyemas.

Figure 3. Presentation of Invasive Pneumococcal diseases.

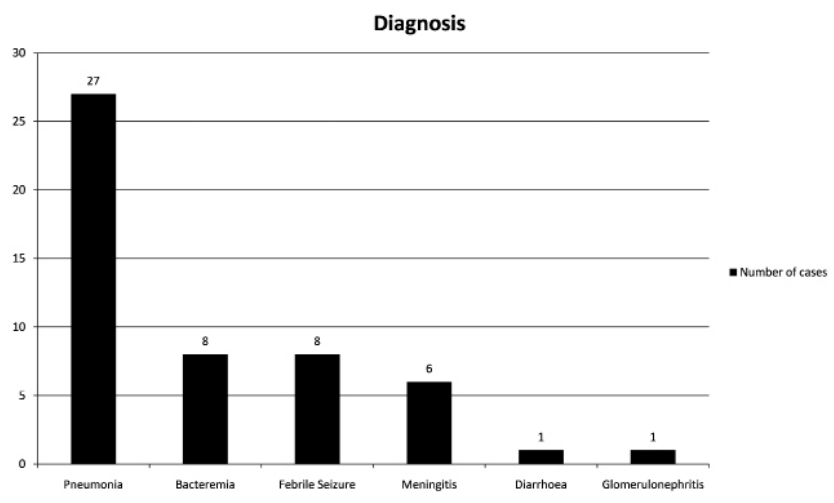


Table 3. Presentation of Invasive Pneumococcal Diseases.

\begin{tabular}{|cc|}
\hline Diagnosis & Number of cases \\
\hline Pneumonia & 27 \\
\hline Bacteremia & 8 \\
\hline Febrile Seizure & 8 \\
\hline Meningitis & 6 \\
\hline Diarrhoea & 1 \\
\hline Glomerulonephritis & 1 \\
\hline
\end{tabular}

\section{TREATMENT}

Most cases were treated with ampicillin (19 cases) or crystalline penicilin (12), some with a third generation cephalosporins, such as cefotaxime (8) or ceftriaxone (5).

In addition to antibiotics, some also received oxygen (18), intravenous fluid (15), antipyretics (21), salbutamol (7) and/ or anticonvulsants (1) One received intercostal thoracic drainage. Two were referred elsewhere, one for a lung abscess and one for biliary atresia.

Thirty patients had fever on admission. With antibiotic treatment fever resolved within 24 hours in 18 patients and within 48 hours in 21 cases.

The majority were successfully discharged;however one child expired.

\section{Mortality}

A two month old male baby was diagnosed with severe sepsis and respiratory failure, pneumococcal meningitis, bilateral acute suppurative otitis media (Staphycoccal) complicated by hypoglycemia and hyperkalaemia. His investigations revealed leukopenia (2000 cells /cm), severe neutropenia (400 cells $/ \mathrm{cm}$ ), hypoglycemia $(18 \mathrm{mg} / \mathrm{dl}$ ), hyperkalaemia $(7 \mathrm{mmol} / \mathrm{L})$ and suspicious cerebrospinal fluid ( $120 \mathrm{WBC} / \mathrm{mm} 3,82 \%$ of neutrophil, $82 \mathrm{mg} / \mathrm{dl}$ of sugar, $120 \mathrm{mg} / \mathrm{dl}$ of protein). Pneumococcus was isolated in the blood and Binax test was positive in the CSF; Staphylococcus aureus grew in pus from ear. The patient expired within 20 hrs of admission.

\section{DISCUSSION}

Indoor hospital invasive pneumococcal disease was found to be more common in children under five (66\%) as found by DD Anh et al in Vietnam, SE Arifeen et.al in rural Bangladesh and R. Batuwanthudawe et al in Sri Lanka. ${ }^{9,10,12}$ SE Arifeen et al in Bangladesh found the incidence more common during infancy. ${ }^{10}$

A history of recent antibiotics was found in only $19 \%$ of our pneumococcal cases; while such history was found by AS Shah et. al and Williams et al in Kathmandu Nepal $(27 \% \& 39 \%) \cdot{ }^{3,4}$ It was upto $68 \%$ in Bangladesh and
Vietnam. ${ }^{8,9}$ Under weight condition is a known risk factor for pneumococcal infection and was present in $43 \%$ of our patients. Samir et.al found $90 \%$ under weight in Bangladesh. ${ }^{6}$ A Naheed found severe malnutrition as one of the risk factors for mortality. ${ }^{8}$

Anaemia was a common finding in 22 cases. Leucocytosis was a found in 27 cases. 13 cases had normal leukocyte counts, showing that a normal leucocyte count does not rule out pnuemococcal infection.

Growth of pneumococci from different body fluids, namely blood, cerebrospinal fluid and pleural fluid were 90\%, 12\% and $7 \%$ of cases respectively, similar to other studies in Kathmandu. ${ }^{3,4}$

Streptococcus pneumoniae was grown in blood in most of the cases of pneumonia and $60 \%$ in pleural fluids from effusion cases. A prospective burden of disease study in Patan Hospital in 2006-2007 by Williams et al reported $9 \%$ clinical meningitis, and 56\% clinical pneumonia (498). Radiologic conformed pneumonia occurred in $40 \%$ (354) and probable or definite meningitis in 5\% (47). S. pneumonia was isolated in $2 \%$ (17) of the children. S.pneumoniae recovery was $0.36 \%$ from blood and $2.44 \%$ from CSF cultures in rural Bangladesh. ${ }^{10}$ Pneumonia topped the list of clinical diagnosis caused by pneumococcal in our study, as it did in AS Shah et al and Williams et al. ${ }^{3,4}$ while meningitis topped the list in A Naheed, SK Shah and AG Faladae. ${ }^{5,6,8}$ Pneumonia was the most common disease followed by bacteremia and meningitis as in William et al. ${ }^{4}$ While meningitis was followed by sepsis and pneumonia in SK Shah, et al. ${ }^{6}$ and by pneumonia and the sepsis in AG Falade.$^{6,8}$

HC Baggett identified a total of 72 culture - confirmed pneumococcal bacteremia cases requiring hospitalization in Thailand, ${ }^{11}$ additional 44 patients had media from positive blood cultures that yielded no growth on subculture but that had positive Binex test.

The Binex test was positive in $83 \%$ of meningitis cases in our study (17\% not tested), close to the $100 \%$ in William et al. ${ }^{4}$

Jeniffer in her multi-site study found that Immuno Chromatographic Test (ICT) such as Binax results were positive for $99 \%(68 / / 69)$ of the culture - confirmed pneumococcal meningitis cases. ${ }^{7}$ By the use of culture and latex agglutination testing (LAT) alone, pneumococci were detected in samples from $7.4 \%$ of meningitis patients in Asia and $15.6 \%$ in Africa. The increased pneumococcal detection by ICT, resulted in similar identification rates across sites, ranging from $16.2 \%$ in Nigeria to $20 \%$ in Bangladesh. ICT detection in specimens from culturenegative cases varied according to region (8.5\% in Africa vs. $18.8 \%$ in Asia; $\mathrm{P}<.001)$, and prior antibiotic use (24.2\% with prior antibiotics use vs. $12.2 \%$ without; $\mathrm{P}<.001)$.

Isolated serotypes were different in different studies. Our most common serotypes were PCV 1, 5, 14. Other studies 
Table 4. Serotypes Isolated in Other Studies. 3,4,5,6,10,12

$\begin{array}{cccccccccccccc}\begin{array}{c}\text { Sero types } \\ \text { isolated }\end{array} & 1 & 2 & 4 & 5 & 7 \mathrm{~F} & 12 \mathrm{~A} & 14 & 15 & 18 \mathrm{C} & 19 \mathrm{~A} & 19 \mathrm{~F} & 22 \mathrm{~F} & 38 \\ \text { no. of studies } & 6 & 3 & 1 & 4 & 2 & 2 & 3 & 1 & 2 & 2 & 1 & 1\end{array}$

Table 2. Serotypes covered by different pneumococcal vaccines.

\begin{tabular}{cc} 
Vaccine & Serotypes covered \\
PCV7 & $4,6 \mathrm{~B}, 9 \mathrm{~V}, 14,18 \mathrm{C}, 19 \mathrm{~F}, 23 \mathrm{~F}$ \\
PCV9 & $4,6 \mathrm{~B}, 9 \mathrm{~V}, 14,18 \mathrm{C}, 19 \mathrm{~F}, 23 \mathrm{~F}, 1,5$ \\
PCV10 & $4,6 \mathrm{~B}, 9 \mathrm{~V}, 14,18 \mathrm{C}, 19 \mathrm{~F}, 23 \mathrm{~F}, 1,5,7 \mathrm{~F}$ \\
\hline PCV13 & $4,6 \mathrm{~B}, 9 \mathrm{~V}, 14,18 \mathrm{C}, 19 \mathrm{~F}, 23 \mathrm{~F}, 1,5,7 \mathrm{~F}, 3,6 \mathrm{~A}, 19 \mathrm{~A}$ \\
PCV23 & $1,2,3,4,5,6 \mathrm{~B}, 7 \mathrm{~F}, 8,9 \mathrm{~N}, 9 \mathrm{~V}, 10 \mathrm{~A}, 12 \mathrm{~F}, 14,15 \mathrm{~B}, 17 \mathrm{~F}, 18 \mathrm{C}, 19 \mathrm{~F}, 20,22,22 \mathrm{~F}, 23 \mathrm{~F}, 33 \mathrm{~F}$
\end{tabular}

also found serotypes such as 7F, 12A, 18C, 19A and 38 which were not found in our study. $3,4,5,6,10,12$

Though penicillin, cefotaxime and ceftriaxone resistance has been identified in the US, our pneumococci are still sensitive to these antibiotics. Continued sensitivity to penicilllin is reported by AG Faladae and Sandra13. AS Shah et. al. showed $4 \%$ of pneumococci were resistant to penicillin. Many of our pneumocci were resistant to cotrimaxozole $(77 \%)$, as reported by many others. ${ }^{3,4,5,6,10,13}$ $\mathrm{R}$ Batuwanti in Srilanka found resistance to 3rd generation cephalosporins to by very high. ${ }^{12}$ Samir K. Shah reported $6 \%$ resistance to chloramphenicol66 but no pneumococcal were resistant in our study.

The coverage of the isolates by PCV 7, 10, 13 and 23 valent vaccine were $36 \%, 54 \%, 54 \%$, and $72 \%$ respectively. However the 23 valent vaccine is not a conjugate vaccine and not suitable below 2 years of age.

Case fatality rate was $2 \%$ in our study while it was $4 \%$ in Bangladesh ${ }^{8}$.

Severe pneumonia, delayed admission are the contributing causes of mortality. In Nigeria mortality in meningitis cases was $37 \%$ while it was $0 \%$ in pneumonia and sepsis cases ${ }^{5}$. We had one mortality, who was a two month old male baby with severe pneumonia and meningitis.

\section{CONCLUSION}

Invasive pneumococcal disease is common among children under five. It largely presents as pneumonia, meningitis and sepsis. Blood, CSF and pleural fluid cultures are still a very important investigation for isolation of Streptococcus pneumonae. The Bines test is a sensitive method of diagnosing $S$ pneumonae infection in CSF. Streptococcus pneumonea in our hospital are still sensitive to penicillin. Chloramphenicol, cefotaxime and erythromycin are equally effective antibiotics. But most isolates are resistance to cotrimoxazole. The 7 valent pneumococcal vaccine would cover $36 \%$ while 10 valent, 13 valent and 23 valent vaccines would cover $54 \%, 54 \%$ and $72 \%$ respectively of the serotypes isolated in our study.

\section{REFERENCES:}

1. Baldehi I, Laurence BE, Secka O, Greenwood B.M. A study of risk factors for Pneumococcal disease among children in a rural area of West Africa. International Journal of Epidemiology. 1996;125:885-893.

2. SF. Dowell, CG. Whitney, C Wright et.al. Seasonal Patterns of Invasive Pneumococcal Disease. International Emerging Infectious Program, Bangkok, Thailand and Centers for Disease Control and Prevention, Atlanta, Georgia USA. 2003,may; Vol.9,No.5.

3. Shah AS, Deloria KM, Sharma PR et al. Invasive Pneumococcal Disease in Kanti Children Hospital, Nepal as observed by South Asian Pneumococcal alliance Network. Clinical Infectious Diseases 2009:48 (Suppl 2). S123-128.

4. Williams EJ, Thorson S, Maskey M et al. Hospital Based Surveillance of Invasive Pneumococcal diseases among young Children in Urban Nepal. S114-122. CID 2009:48 (Suppl 2).

5. AG Faladae. Invasive Pneumococcal Disease in Children Aged $<5$ years Admitted to 3 Urban Hospitals in Ibadan, Nigeria. S190-196. CID 2009:48 (suppl 2).

6. SK Shah, A Naheed, SE Arifeen, M Isla et.al. and Pneumococcal Study Group. Surveillance for Invasive Streptococcus Pneumoniae Disease among Hospitalized Children in Bangladesh, Antimicrobial susceptibility and Serotype Distribution. CID 2009:48 (Suppl 2) S75-S81.

7. JC. Moisi, SK. Saha, AG. Falade, BMN Lafourcade et.al. Enhaced diagnosis of Pneumococcal Meningitis with Use of the Binax NOW Immunochromatographic Test of Streptococcus pneumonia antigen: A Multisite Study. Clinical Infectious Disease 2009:48(Suppl 2) S49-S56.

8. A Naheed, SK.Saha, RF. Breiman et.al. Multi Hospital Surveillance of Pneumonia Burden among Children $<5$ years Hospitalized for Pneumonia in Bangladesh. Clinical Infectious Disease 2009:48 (Suppl 2) S82-S89.

9. DD Anh, PE. Kilgore, MPE. Slack et.al. Surveillance of Pneumococcal -associated disease among Hospitalized children in Khanh Hoa Province, CID 2009:48 (Suppl 2) Vietnam. S 57-S64.S.

10. SE. Arifeen, SK. Saha, S Rahma et.al. Invasive Pneumococcal Disease among children in rural Bangladesh: Results from a Population Based Surveillance. CID 2009:48 (Suppl 2) S103-S113.

11. HC. Baggett, LF. Peruski, SJ. Disen et.al. Incidence of Pneumococcal Bacteremia Requiring Hospitalization in rural Thailand. CID 2009:48 (Suppl 2) S65-S74.

12. R. Batuwanthudawe, K. Karunathne, M. Dasanayake et.al. Surveilance of invasive Pneumococcal Disease in Colombo, Sri Lanka. CID 2009:48 (Suppl 2) S136-S146.

13. SMudhune and M Wamae. Reports on Invasive Disease and Meningitis due to Hemophilus influenza and Streptococcus pneumonia from Network of surveilance for Pneumococcal Disease in East African Region. CID 2009:48 (Suppl 2) S147-S152. 